\title{
Reformulation et textualité
}

\section{dans les contes de La Maison Tellier de Maupassant}

\author{
Magri-Mourgues, Véronique \\ Université de Nice Sophia-Antipolis, UMR 7320, Bases, Corpus et Langage \\ magri@unice.fr
}

\section{Introduction}

Le terme de reformulation est ambivalent : il peut en effet désigner à la fois l'opération de sémantique discursive qui crée «une variante paraphrastique d'un segment linguistique » (F. Neveu 2004: 251), et l'objet linguistique qui résulte de cette opération. Cette ambivalence définitionnelle induit une double approche de la reformulation. Syntaxique et sémantique d'abord, puisqu'elle suppose l'inscription de deux unités dans la chaîne linéaire du texte, une formulation première, qui fait office de support, une re-formulation, apport sémantique par rapport à la précédente. Par ce préfixe itératif, elle se donne comme unité charnière articulant un avant-texte auquel elle renvoie anaphoriquement et une expression nouvelle qu'elle pointe comme aboutissement d'un processus discursif. L'autre approche de la reformulation est pragmatique parce que se pose la question de l'acte de langage réalisé par cette mise en relation syntagmatique et que l'analyse est relancée par les enjeux communicationnels d'un tel processus ainsi que par sa finalité discursive en contexte.

En assurant le passage d'une unité à l'autre, «la reformulation est un facteur de textualité » (J.-M. Adam $1990: 172)$; celle-ci pouvant se définir comme " un équilibre délicat entre une continuité-répétition d'une part, et une progression de l'information, d'autre part» (J.-M. Adam 1990 : 45). Ce pourrait être une définition générale de la reformulation qui se situe entre deux extrêmes, la répétition redondante d'une part et la succession apparemment incohérente d'unités d'autre part.

L'approche syntaxique de la reformulation s'intéresse aux différents modes de liage qui tissent la trame textuelle; l'approche sémantique et pragmatique analyse le rapport de sens à établir entre les unités et la motivation de la reformulation dans une conception interactionnelle de l'énoncé. Une approche cognitive aurait à établir le rapport instauré entre l'unité reformulée et le référent, comme indicateur de la perception du réel et de sa catégorisation.

Les configurations syntaxiques sont elles-mêmes diverses et se distinguent en surface par la présence ou non d'un marqueur. Les marqueurs linguistiques de reformulation ont fait l'objet d'inventaires qui ont établi des paradigmes fixés et classifiés en vertu de spécificités syntaxiques, morphologiques ou sémantiques; de nombreuses études se concentrent par exemple sur les marqueurs formés sur le verbe « dire » ${ }^{1}$. Le corpus d'étude est constitué des contes de la Maison Tellier de Maupassant : la structure d'un recueil de contes peut être envisagée elle-même comme une illustration d'un processus 
de reformulation; chaque conte est un fragment d'un ensemble dont le lecteur doit reconstituer la cohérence et l'unité en évaluant l'équilibre entre points communs, glissements et divergences d'un conte à l'autre. Un conte est, chaque fois, différent du précédent et, cependant, la réunion en recueil de plusieurs d'entre eux revendique des affinités. La cellule macrostructurale du recueil repose sur ce principe de textualité, défini par la tension entre continuité et renouvellement.

Ces contes de Maupassant sont d'autant plus un corpus de choix pour le sujet qui occupe cet article qu'ils présentent une récurrence particulière au niveau microstructural cette fois. Dans ces contes, en effet, un procédé de construction phrastique récurrent complexifie et, en même temps, peut servir à affiner les analyses qui portent sur le processus de reformulation ${ }^{2}$. Il s'agit de la parataxe asyndétique qui juxtapose des unités dont la relation est à rétablir - en l'absence de tout ligateur explicité - par inférence. Se justifiant de la fréquence de ce procédé dans le corpus d'étude, on étudiera la reformulation lorsqu'elle se réalise sans marqueur explicite. Se pose alors la question préalable de l'identification de ce processus en l'absence de signaux univoques puis de son rôle dans la constitution d' "un tout cohésif et cohérent, c'est-à-dire un TEXTE » (J.-M. Adam 1990 : 49), perçu comme « tout signifiant» (J.-M. Adam 1990 : 33). C'est d'abord la mise en texte ou textualisation de la reformulation qui sera abordée, à la fois au travers de ses relations avec la référenciation et des marqueurs linguistiques explicites qui l'inscrivent dans la linéarité textuelle. Sur le plan sémantique, la reformulation est envisagée, dans les études déjà réalisées, comme un processus fondé sur l'équivalence sémantique; cette théorie sera interrogée ensuite pour en tester la validité dans le corpus d'étude et en observer le fonctionnement au niveau de l'organisation phrastique. La juxtaposition pose les unités, données comme équivalentes, dans une continuité linéaire ; dès lors, c'est l'ordre même de ces unités qui doit être problématisé pour en étudier l'incidence dans la progression du texte et dans la constitution des configurations discursives.

\section{Reformulation et textualisation - la lecture linéaire du texte}

\subsection{Reformulation et référenciation}

L'approche cognitive et donc la relation au référent est nécessaire pour établir la réalité de l'opération de reformulation. En sémantique logique, le sens se définit comme relation de dénotation entre des signes linguistiques et leurs référents ${ }^{3}$; la sémantique linguistique pose le sens « comme une relation linguistique entre des signifiés » (Voir F. Neveu 2004 : 263), ce qui permet de distinguer d'emblée désignation et signification.

L'unicité référentielle des expressions nominales en relation de reformulation est un premier critère de reconnaissance. Un même objet du monde ou une classe d'objets est désigné(e) par plusieurs dénominations, sans qu'il soit exclu que ces dénominations entretiennent des rapports paraphrastiques entre elles, autrement dit, que leur signifié présente des points de convergence sémantique - question traitée dans la seconde partie. Ce critère permet d'exclure du champ d'étude des structures juxtaposées comme dans l'énumération additive, caractérisée par des désignations successives de référents 
différents et non pas par multi-dénomination du même référent. De fait, et pour autant qu'on puisse supposer que l'autonomie syntaxique signifiée par la juxtaposition puisse induire une autonomie sémantique des unités contiguës, on écartera a priori les exemples où une conjonction «et» peut être virtuellement rétablie, dans son sens d'addition pure. La coordination, dans cet emploi, accorde une égalité de statut et de rôle aux unités reliées, sans établir une quelconque hiérarchie entre elles. Si on postule l'indépendance sémantique des unités, on ne peut parler de reformulation qui repose anaphoriquement sur l'unité située à sa gauche. On est alors dans le cas d'un processus d'addition, d'accumulation d'items, non liés l'un à l'autre.

C'est une disposition spécifique des séquences descriptives dont "l'énumération apparaît [...] comme une sorte de base ou de degré zéro » (J.-M. Adam 2001 : 81). L’inventaire descriptif s'appuie sur des dispositifs syntaxiques sériels.

\section{[1] Elle avait les joues rouges et pleines, une large poitrine saillante sous l'indienne de son caraco, de grosses lèvres fraiches, et sa gorge, presque nue, était semée de petites gouttes de sueur. (« Histoire d'une fille de ferme » : 83)}

L'ordre linéaire des trois premiers syntagmes compléments d'objet n'est pas contraint syntaxiquement et rien n'empêche d'imaginer un agencement alternatif ${ }^{4}$. Cette possibilité théorique montre que les unités sont autonomes aussi sur le plan sémantique. Lorsque l'énumération concerne des prédicats verbaux, elle sert la progression narrative. Nulle équivalence sémantique ne lie les unités juxtaposées mais une structuration spatio-temporelle se réalise au fil des syntagmes verbaux, étayée par la présence de l'adverbe « enfin » qui joue le rôle d'organisateur temporel dans l'exemple $[2]$.

[2] Et, sous l'impulsion des longues rames recourbées, les yoles rapides glissaient sur la rivière, s'éloignaient, diminuaient, disparaissaient enfin sous l'autre pont. (« La Femme de Paul » : 164)

Si le référent doit donc être unique pour que le lecteur puisse lire une opération de reformulation entre deux unités juxtaposées, l'opération de référenciation, elle, peut être double ou même plurielle. Intéressons-nous pour cette démonstration aux cas où deux syntagmes nominaux sont juxtaposés.

Les syntagmes nominaux ainsi rapprochés peuvent être actualisés par le même type de déterminants; ce sont les cas les plus nombreux du corpus d'étude (M. Noailly 2000), en particulier ceux qui emploient des démonstratifs.

[3] Il subissait cet ensorcellement féminin, mystérieux et tout-puissant, cette force inconnue, cette domination prodigieuse, venue on ne sait d'où, du démon de la chair, et qui jette l'homme le plus sensé aux pieds d'une fille quelconque. (« La Femme de Paul »: 173) $)^{5}$

Les trois syntagmes «cet ensorcellement féminin », "cette force inconnue », «cette domination prodigieuse " peuvent chacun suffire seul à la complétude de l'énoncé, sans qu'une relation syntaxique hiérarchique s'établisse entre eux. On ne peut en effet restaurer une relation prédicative explicite entre les termes; il serait difficile par exemple de gloser l'énoncé par * «cet ensorcellement féminin est cette force inconnue »; l'énoncé acceptable serait en effet «cet ensorcellement féminin est une 
force inconnue »; ce cas de figure est bien explicité par M. Noailly (M. Noailly 2000 : 53) : le parallélisme des structures juxtaposées, induit par l'identité des déterminants dans les structures juxtaposées, bloque la relation appositive éventuelle. Le référent est unique mais des désignations successives du même référent sont assurées par les groupes nominaux démonstratifs. C'est une structure qui peut s'interpréter comme un cas de «coordination (ouverte)», a fortiori quand on constate, comme dans cet exemple, plus de deux groupes nominaux juxtaposés.

Un autre exemple est particulièrement intéressant en ce qu'il manifeste cette fois l'hétérogénéité des déterminants ; on a le schéma ternaire [le N, ce N/ un N].

[4] Alors commença le délire, ce délire fuyant des gens de la campagne qui se croient frappés par un sort, un besoin fou de partir, de s'échapper, de courir devant le malheur comme un vaisseau devant la tempête. («Histoire d'une fille de ferme » : 91)

Le lien établi entre les trois groupes peut s'interpréter, sur le plan sémantique, en termes de reformulation mais la variation du déterminant dans les groupes juxtaposés leur confère un statut reformulatif différent, qui croise des critères d'appréciation d'ordre syntaxique et sémantique ; le non-accord du verbe antéposé au pluriel plaide en faveur d'un référent unique pour les trois syntagmes successifs. Cependant, la variété du déterminant induit des différences quant à la capacité de référenciation de chacun d'eux et, corollairement, quant à la relation syntaxique instaurée entre eux. Celle-ci peut être cumulative ou hiérarchique, selon que la reformulation repose sur une simple juxtaposition ou sur une relation de type prédicatif ou appositif. L'ordre des unités est contraint - on ne pourrait accepter la suite *ce délire..., le délire dans ce cotexte particulier du fait sans doute aussi de la valeur anaphorique du syntagme démonstratif ce qui règle le rôle de chacun des syntagmes : «le délire » est le terme support et « ce délire fuyant des gens de la campagne... » intervient comme refomulation explicitante et expansive du syntagme nominal «le délire», doté d'un article défini de notoriété. Néanmoins, ce syntagme démonstratif conserve une capacité référentielle potentielle : il pourrait en effet suffire seul à la complétude de l'énoncé, indépendamment du premier syntagme, là encore en vertu du rôle déictique que le syntagme démonstratif recouvrerait alors complètement. Dès lors, c'est la relation syntaxique entre les deux éléments qui se trouve problématisée. La fonction référentielle, qui peut être définie comme « une relation qui unit une expression linguistique en emploi dans un énoncé avec l'objet du monde qui se trouve désigné par cette expression » (F. Neveu 2004 : 250-251), peut s'éluder au profit de la fonction appositive. L'apposition peut être définie comme «l'association, plus ou moins paradoxale, d'une relative indépendance syntaxique, marquée par le détachement, et d'une non-autonomie référentielle » $(\mathrm{M}$. Noailly 2000 : 46-59). Le statut appositif pourra être attribué à un groupe nominal s'il est dépourvu de fonction référentielle directe et s'il fonctionne comme un prédicable d'un autre groupe support qui assure la fonction de référenciation. Dans cette perspective, "le délire » est le terme support et « ce délire...» fonctionne comme le terme apport, construit en prédication seconde. On peut cependant hésiter entre un processus de reformulation par apposition ou par simple juxtaposition, qui recatégorise ou reclassifie, mais ne relève pas du système appositif, quand le second syntagme conserve sa pleine capacité référentielle. 
Le troisième syntagme " un besoin fou de partir » est à placer à un autre niveau que le second. Là encore, son ajout ne modifie par l'accord du verbe initial, ce qui argumente la relation de dépendance qu'il entretient avec un autre élément de la phrase. De plus, « un besoin fou » n'est pas attendu comme sujet d'un verbe comme « commencer »; ce qui prive ce syntagme de toute capacité de référenciation et d'autonomie syntaxique. «Un besoin fou » ne réfère pas directement mais par le biais d'un syntagme nominal précédent, sans que l'on puisse décider s'il s'agit du premier ou du second des groupes situés à sa gauche. Le syntagme «un besoin fou de partir» se trouve dépourvu de fonction référentielle; une relation de type appositif ou attributif peut en revanche être rétablie avec l'un des deux syntagmes précédents. Ce troisième syntagme joue un rôle définitionnnel sur le plan sémantique et, syntaxiquement, une relation hiérarchique ordonnée est instaurée.

Le second syntagme qui reprend le substantif « délire » garde une potentielle valeur de désignation d'un référent unique tandis que le dernier, par l'emploi du déterminant indéfini «un», s'oriente davantage vers le statut de segment métalinguistique, explicitation ou glose du mot même de " délire ». Une preuve en est qu'un marqueur du type « autrement dit » pourrait introduire ce dernier élément alors que celui-ci n'est pas acceptable comme possible introducteur du second. Selon certains linguistes, la glose et la reformulation se distingueraient par leur statut pragmatique : la première relevant de l'épilinguistique, est un commentaire sur le dire, la seconde est une autre manière de dire $^{6}$. Je ne reprendrai pas ces distinctions, quitte à établir une sous-catégorisation qui décline divers types de reformulation, comme la reformulation métalinguistique sans potentiel de référenciation par opposition à celle qui permet une nouvelle dénomination.

L'unicité du référent est un critère de la reformulation qui est quelquefois appuyé par des indices explicites fonctionnant comme indices instructionnels compensatoires à l'absence de marqueurs explicites. Contraint par la linéarité obligée du texte, le lecteur est sensible au retour du même dans la phrase qui le conduit à une lecture à la fois cursive et analogique. C'est ce que nous allons observer dans le point suivant.

\subsection{Reformulation et connexion syntagmatique}

Un indice compensatoire à l'absence de marqueur de reformulation peut être la reprise de la même préposition en structure parallèle : le parallélisme syntaxique induit a priori une équivalence fonctionnelle des unités. Le procédé est récurrent dans le corpus reposant sur des structures en anaphore au sens rhétorique du terme. La contiguïté dans la linéarité discursive induit un rapprochement sémantique des unités qui renvoient au même référent. Les exemples [5] à [7] sont réunis uniquement en raison de leur similarité structurelle :

[5] Une espèce de communion avec cette splendeur calme du soir, avec ce vague et mystérieux frisson de vie épandue, avec cette poésie pénétrante, mélancolique. ( $« \mathrm{La}$ Femme de Paul » : 175)

La reprise d'un mot, ici la préposition « avec », donne un rythme volontiers ternaire aux phrases de Maupassant. Dans ce cas de figure, il paraît difficile de lire une structure 
appositive entre les syntagmes juxtaposés au motif qu'une relation prédicative ne peut être établie entre eux; il s'agit plutôt d'une «coordination (ouverte) » (M. Noailly 2000 : 53). Les trois syntagmes démonstratifs sont trois formulations successives du même référent ; un parallélisme syntaxique s'établit entre eux qui propose une phrase à étagement.

La reformulation peut encore s'appuyer sur la reprise du même substantif complété par un prédicat ou modulé par des prédicats différents.

[6] Dans son regard fuyant mille choses m'apparurent, mille choses ignorées jusqu'ici. («Au printemps »: 157)

L'ajout du participe de valeur adjectivale «ignorées » explicite le syntagme nominal précédent. La reprise expansée du même nom apporte une précision corrective au syntagme précédent, tout en conservant une capacité de référenciation. La reformulation joue un rôle caractérisant; sur le plan syntaxique, il n'y a pas de relation appositive mais simple juxtaposition : on ne peut en effet rétablir une relation prédicative entre les deux syntagmes qui conservent une autonomie référentielle, réalisée ou latente pour la seconde occurrence. La nouvelle caractérisation permet une nouvelle catégorisation par juxtaposition.

[7] Elle lui lança ce regard énigmatique, ce regard à perfidies qui apparaît si vite au fond de l'œil de la femme. (« La Femme de Paul» : 176)

La relative en facteur commun aux deux antécédents juxtaposés est un indice de la reformulation, à cause de la présence du déterminant démonstratif qui confère au syntagme une portée cataphorique. Une glose par « ou plutôt » pourrait expliciter le lien sémantique entre les syntagmes nominaux successifs. La deuxième occurrrence se substitue à la précédente dans un souci d'ajustement des mots aux choses qui juxtapose des rectifications successives; ou bien ce peut être une structure où les dénominations s'empilent (M. Noailly 2000 : 54) sans se remplacer pour donner une vision plurielle du référent; les dénominations se multiplient, évitant la description monolithique et ouvrant, au contraire, à des représentations diverses qui misent sur la relativité des perceptions.

En l'absence de marqueur, d'autres indices fonctionnent comme indices de l'opération langagière de la reformulation, le parallélisme syntaxique et rythmique, la reprise du même terme dont le sémantisme est explicité ou modulé, tandis qu'un même référent est conservé même si le processus de référenciation, quant à lui, peut être redoublé. La reformulation s'inscrit dans la linéarité textuelle. Les parallélismes syntaxiques amorcent une construction phrastique à étagement. La proximité sémantique plus ou moins grande entre les unités favorise une lecture tabulaire ou verticale, par la juxtaposition d'expressions données comme équivalentes. Celle-ci freine la lecture linéaire en proposant plusieurs syntagmes pour un même poste syntaxique. 


\section{Reformulation et équivalence sémantique - la lecture tabulaire du texte}

Le TLFi définit la reformulation comme une " nouvelle formulation qui reproduit autrement ce qui a déjà été exprimé ». Cette définition sommaire recèle déjà tout le paradoxe de la reformulation, comme activité langagière, tendue entre la répétition du même et la création d'un autre énoncé. Des linguistes ont résumé cette ambivalence en observant que ce processus « dans le même temps qu'il pose un dit nouveau, re-dit un propos antérieur » (Le Bot et alii 2008 : 11). La reconnaissance de ce propos antérieur, en dehors d'une répétition pure et simple de la forme même de l'énoncé, doit se fonder sur la reconnaissance d'une parenté sémantique entre les énoncés, voire d'une homologie ou équivalence sémantique, par-delà des transformations éventuelles de surface. Cette reconnaissance d'abord intuitive repose sur la compétence du récepteurlecteur, à même d'identifier un invariant de sens par-delà les transformations formelles de surface ; elle relève de l'interprétation du récepteur et met en jeu une dialectique du même et de l'autre.

De fait, le processus de la reformulation voisine avec des procédures discursives qui reposent sur l'établissement d'une relation d'équivalence sémantique entre deux unités, notamment la paraphrase ${ }^{7}$, envisagée comme traduction intralinguale (R. Martin 1976 : 77) ou comme « une relation d'équivalence entre deux énoncés, l'un pouvant être la reformulation ou non de l'autre $»^{8}$. Entre ces deux opérations de sémantique discursive, reformulation et paraphrase, il n'y a pas recouvrement mais points de contact.

D'une part, paraphrase et reformulation ne se réalisent pas aux mêmes niveaux; le niveau minimal où peut s'exercer la relation paraphrastique est en effet, selon certains linguistes, celui de la phrase :

\footnotetext{
Une relation de paraphrase ne peut en effet être posée qu'entre des unités constituant une prédication susceptible de supporter une assertion. (C. Fuchs $1994: 45$ )
}

On s'intéressera aussi cependant au niveau inférieur de la phrase, syntagmes ou lexèmes. L'association par simple juxtaposition peut en effet affecter différentes unités et établir un rapport de reformulation paraphrastique ; la relation ne s'établit pas cependant de manière identique selon que ces items sont des unités phrastiques ou des unités inférieures à la phrase, comme des noms, des adjectifs, des verbes ou des adverbes. Tous ces cas de figure sont représentés dans le corpus d'étude choisi, quoique dans des proportions inégales.

D'autre part, ces deux opérations ne sont pas liées par une relation de symétrie : la reformulation n'est pas forcément paraphrastique. Les travaux sur la paraphrase ont établi une typologie qui distingue trois types de paraphrase : la paraphrase linguistique, pragmatique et référentielle 9 . Les deux derniers types nécessitent de connaître la référence des termes ou la situation extra-linguistique pour décider du caractère paraphrastique du lien entre énoncés; pour l'étude qui m'occupe, c'est au premier type de paraphrase dans son rapport à la reformulation que je m'intéresserai, sans exclure cependant sa possible dimension énonciative. 


\subsection{Reformulation paraphrastique - la thèse de l'équivalence sémantique ${ }^{10}$}

La notion d'équivalence sémantique se distingue de l'identité de sens purement théorique pour établir un invariant sémantique, un noyau sémantique prégnant, qui s'impose en éludant les différences sémantiques périphériques. On retrouve les principes de la grammaire générative qui distingue structure profonde et de surface et qui, à partir d'un modèle minimal de base, peut prévoir au gré de transformations syntaxiques, une série de variantes phrastiques susceptibles d'être appréhendées en termes d'équivalence sémantique.

Les phrases en relation de paraphrase partagent, à un certain niveau, un même sémantisme de base, tout en se différenciant sémantiquement à d'autres niveaux ${ }^{11}$.

Dans l'optique de la sémantique vériconditionnelle, le critère pour établir l'équivalence sémantique est celui de l'identité des valeurs de vérité.

\footnotetext{
Deux phrases Pi et Pj seront donc dites en relation de paraphrase linguistique si, pour tout locuteur et en toute situation, $\mathrm{Pi}$ est logiquement équivalente à $\mathrm{Pj}$. [...]

Cela revient à dire que si l'une des deux phrases est vraie, l'autre est nécessairement vraie et si l'une est fausse, l'autre est nécessairement fausse. (R. Martin 1976 : 85)
}

La mise en relation paratactique de plusieurs unités phrastiques peut être interprétée en termes de reformulation; observons l'exemple suivant :

[8] La rivière est silencieuse et perfide. Elle ne gronde pas, elle coule toujours sans bruit. («Sur l'eau » : 73)

Un lecteur peut intuitivement percevoir l'homogénéité lexicale de ces deux phrases: différentes parties de discours rejoignent le même champ lexical; l'adjectif « silencieuse » d'abord puis les syntagmes verbaux « ne gronde pas, coule sans bruit », qui développent tous l'idée du silence. La succession linéaire des trois syntagmes verbaux et la ponctuation forte qui sépare les deux phrases plaident en faveur d'une seconde phrase à fonction explicative et non pas d'une reformulation paraphrastique; le sens de "perfide» ne se trouve pas réitéré de manière explicite dans les segments suivants; c'est seulement à titre de connotation que le silence peut être associé à la perfidie. Entre les deux segments juxtaposés suivants, en revanche, la relation est plus nettement de reformulation paraphrastique. Le sens logique de elle ne gronde pas et elle coule toujours sans bruit est équivalent par un processus de substitution lexicogrammaticale : on peut proposer cette équivalence si on considère que "coule », prédicat-type de « rivière », voit ainsi son apport informatif neutralisé au bénéfice de la locution adverbiale «sans bruit» qui rejoint le sémantisme de « elle ne gronde pas »; de façon symétrique, une rivière ne pourrait «gronder » qu'en coulant. Le seul apport informatif véritablement nouveau, dans cette hypothèse, est celui véhiculé par l'adverbe « toujours ».

Dans l'exemple suivant, l'équivalence sémantique entre énoncés est doublée d'une variation de registres :

[9] Son mari aussi vieillissait, " se mangeait les sangs », disait-on, se consumait en espoirs inutiles. («Histoire d'une fille de ferme » : 99) 
Ce cas se rapproche de l'exercice de la traduction, qui repose sur l'interprétation d'un énoncé-source et sur sa reformulation en un énoncé-cible appartenant à un autre univers langagier. En l'occurrence, le segment entre guillemets dans le texte se démarque du reste de l'énoncé comme fragment de discours rapporté, clairement identifié comme tel par l'incise "disait-on». C'est un cas de modalisation autonymique, décrit par J. Authier-Revuz, qui associe un emploi simultané en usage et en mention de l'expression; l'expression populaire se trouve traduite et commentée par le segment suivant qui se trouve à la fois en situation d'explicitation et de contraste. Les pluriels de part et d'autre assurent une homogénéisation formelle des segments tandis que le verbe métaphorique « se consumait » introduit une discordance de registre, indice de l'énoncé polyphonique.

L'équivalence sémantique entre deux énoncés rejoint la question de la synonymie qui peut être évaluée entre phrases et entre unités lexicales et croise les relations de sémantique externe qui structurent le lexique comme la synonymie, l'hyperonymie et la métonymie, susceptibles de définir les relations sémantiques établies entre les deux unités.

La relation synonymique est depuis longtemps démontrée par l'évaluation des commutations possibles dans divers énoncés: les éventuelles limitations distributionnelles des lexèmes permettent de faire la part entre les traits sémantiques communs d'un côté et différentiels de l'autre. En langue, elle peut être explicitée en termes de traits dénotatifs et connotatifs. Les linguistes ${ }^{12}$ se rejoignent pour créditer les sèmes dénotatifs d'une certaine stabilité et de leur connaissance partagée au sein d'une communauté :

Les sèmes dénotatifs [...] déterminent d'une façon stable et avec une vaste assise sociale la signification. (R. Martin 1976 : 91)

L'interprétation de deux énoncés en termes de reformulation s'appuie sur les synonymes en langue, autrement dit, identifiés par les dictionnaires.

[10] Il semblait nourrir une inquiétude, porter en lui un souci. (« Histoire d'une fille de ferme $»: 96)$

Cet exemple établit une équivalence entre deux expressions, "nourrir une inquiétude », "porter en lui un souci », encouragée syntaxiquement par la mise en facteur commun du sujet et du verbe recteur. "Inquiétude » et " souci » sont donnés comme synonymes en langue par le TLFi, ce qui fait d'eux des termes théoriquement interchangeables dans un grand nombre de contextes ${ }^{13}$.

\footnotetext{
On dira de deux morphèmes, de deux mots, de deux syntagmes, plus généralement de deux unités $\mathrm{a}$ et $\mathrm{b}$ qu'ils sont synonymes, si la substitution de $\mathrm{a}$ à $\mathrm{b}$ dans $\mathrm{p}$ conduit à une paraphrase q de p. (R. Martin $1976: 113$ )
}

Ces relations synonymiques virtuelles en langue sont actualisées en discours et, du fait de l'équivalence sémantique globale que le lecteur perçoit, peuvent induire une relation de reformulation paraphrastique.

Les adjectifs juxtaposés fonctionnent comme les prédicats d'un même nom support dans l'exemple suivant : 
Le TLFi donne pour synonyme d' « officiel », patent, et pour l'adverbe « patemment », formé sur patent, "publiquement ». Un lien d'équivalence en langue est reconnu entre ces trois adjectifs que l'énoncé exemplifie par cette succession ternaire.

Les dénominations plurielles du même référent situent la règle d'équivalence entre langue et discours :

[12] - Ah ! Tu l'avoues donc, gueuse ! Et qu'est-ce que c'est, ce merle-là ? Un va-nupied, un sans-le-sou, un couche-dehors, un crève-la-faim? (« Histoire d'une fille de ferme » : 93)

La succession des unités est perçue par le lecteur comme l'expression d'items équivalents pour désigner une personne misérable et pitoyable. Leur appartenance partagée au domaine familier accentue le parallélisme.

L'équivalence sémantique peut être instaurée plus clairement en discours. C'est le cas en particulier lorsque les suites sont hétérogènes sur le plan syntaxique ; la comparaison entre deux exemples est éclairante :

\footnotetext{
[13] Il éprouva de nouveau un soulagement, un repos, une tranquillité subite. («En famille » : 117)

[14] Elle voulait la paix, le repos complet, dormir sans fin. («Histoire d'une fille de ferme » : 92)
}

Dans l'exemple [13], le lecteur perçoit la proximité sémantique des trois substantifs. Il suffit de lire les rubriques de dictionnaires pour retrouver ceux-ci en effet associés dans leur définition respective. En revanche, dans l'exemple [14], l'hétérogénéité des unités successives mise sur une équivalence plus clairement établie en discours. Un paradigme se construit en discours à réévaluer selon le contexte particulier de la nouvelle.

L'équivalence construite trouve une parfaite illustration dans la reformulation métaphorique, comme dans cet exemple :

[15] Toutes ses ruses sont tendues, toutes ses armes aiguisées, toutes ses perfidies préparées. («Au printemps »: 158)

Le parallélisme syntaxique des trois groupes en parataxe induit une équivalence qui peut être récupérée sur le plan du sens. Les mots « ruse » et "perfidie » sont donnés comme synonymes par le TLFi; de la même façon, les participes en emploi adjectival, « tendues » et " préparées », expriment une même tension prospective. Seul le segment central se distingue, non pas par le sens globalement équivalent encore, mais par sa portée métaphorique. La succession de ces syntagmes reflète un regard différent sur une même réalité, un point de vue changeant. C'est là un cas frontière entre la reformulation paraphrastique et celle qui ne l'est pas: le mot «arme »n'est pas donné en langue comme synonyme de « ruse » ou de «perfidie». Tout au plus, si l'image peut être considérée comme un lieu commun, pourrait-on parler d'une synonymie acquise ou connotée. Pour Jakobson, l'expression métaphorique appartient au plan paradigmatique, au même titre que les unités métalinguistiques ${ }^{14}$; une équivalence paradigmatique s'institue, parfois soulignée par un modalisateur, tel « comme »:

[16] D'une langueur tendre, d'un bonheur léger, comme d'une vapeur de bien-être.

(«La Femme de Paul »: 175) 
La reformulation ne peut plus être dite paraphrastique lorsque les énoncés successifs, tout en renvoyant à un référent unique, ne partagent pas de sèmes en langue.

\subsection{Reformulation non paraphrastique}

Cette fois, «c'est [...] la stabilité du référent qui fonde le noyau sémantique commun tandis que la diversité des points de vue donne naissance aux sémantismes différentiels » (C. Fuchs 1982a : 56). L'exemple suivant est un exemple où se manifeste un jeu sur les points de vue :

[17] On eût dit que ce peuple, ce ramassis de corrompus, saluait un chef. ( « La Femme de Paul »: 170)

Le second syntagme «ce ramassis de corrompus» propose une réorientation énonciative de la désignation. Il émet un jugement du locuteur au travers de termes connotés négativement. La reformulation s'appuie sur un changement de tonalité, depuis un terme neutre jusqu'à une expression périphrastique évaluative. L'ordre des deux énoncés est contraint par ce mouvement esquissé de la neutralité vers la subjectivité. Deux désignations successives du même référent sont assurées par le biais des syntagmes avec déterminant démonstratif de valeur déictique. Le second active des traits connotatifs du mot «peuple», des traits péjoratifs virtuels et latents ${ }^{15}$. Le même type de réorientation énonciative est à l'œuvre dans l'exemple suivant :

[18] Heureuses des regards fixés sur elles, supérieures à cette foule, à cette tourbe, à cette plèbe. («La Femme de Paul»: 172)

«Cette foule, cette tourbe, cette plèbe » renvoient successivement au même référent. C'est le point de vue des femmes qui s'exprime, dans une stratégie de bivocalité ou de superposition des voix énonciatives.

La reformulation, qu'elle soit paraphrastique ou non, table sur une équivalence sémantique en langue, réactivée par le discours, ou bien construite en discours. Ce faisant, elle surimpose à l'organisation linéaire de la phrase, une lecture tabulaire du texte ou verticale par les choix paradigmatiques proposés qui viennent s'empiler et freiner la lecture cursive tout en en déployant les virtualités linguistiques.

\section{Reformulation et configurations discursives - la lecture réticulaire et spéculaire du texte}

\subsection{Orientation discursive}

La séquentialité problématise la relation entre les unités qui visent le même référent et qui sont liées par une équivalence sémantique. Ces items successifs construisent une série ordonnée dont l'ordre peut être supposé non aléatoire. Si l'équivalence sémantique peut être démontrée, par quoi peut être déterminé l'agencement de ces unités, de façon que le texte progresse et ne piétine pas?

Pour être interprétée comme un texte, une suite d'énoncés doit donc [...] non seulement apparaître comme une séquence d'unités liées, mais aussi comme une séquence progressant vers une fin. (J.-M. Adam $1990: 46$ ) 
L'orientation discursive des unités juxtaposées peut reposer sur une relation hiérarchique d'hyperonyme à hyponyme.

[19] Pris d'un respect, d'une affection tout autre, d'un commencement d'amour vrai.

(«Histoire d'une fille de ferme »: 82)

Le binôme "affection » et "commencement d'amour vrai » repose sur un rapport d'inclusion sémantique, l'amour étant un exemple d'affection, un des hyponymes d' « affection ». L'ordre des éléments est cette fois dicté par la langue et tient sans doute aux structures implicatives du lexique (R. Martin 1976: 43-45) qui imposent une orientation.

Un autre mouvement s'esquisse dans le corpus qui adopte une démarche inductive, du particulier au général :

[20] Il eut un sourire lamentable, un de ces sourires dont on voile les plus horribles souffrances. (La Femme de Paul : 176)

A. Vial déjà, notait « une sorte de tic » (A. Vial $1954: 603$ ) chez Maupassant dans la suite généralisante « un de ces + nom + relative ». Avec le présent de l'indicatif dans la relative subséquente, une dynamique généralisante est à l'œuvre qui fait passer de l'expérience ponctuelle notée par le déterminant indéfini à valeur particularisante à une vérité générale qui en appelle à la connivence avec le lecteur. Ce cas est analysé comme exophore mémorielle ${ }^{16}$.

La succession dans la chaîne linéaire orientée de la phrase laisse supposer une progression qui peut être interprétée comme gradation. La reformulation s'appuie sur une suite scalaire étayée notamment par la présence d'un adverbe comme " presque » qui signale une tension vers un sémème non complètement réalisé en discours, vers lequel l'énoncé tend dans un mouvement asymptotique :

[21] Il avait sept ou huit ans. Il était un peu pâlot, très propre, avec l'air timide, presque gauche. («Le papa de Simon »: 133)

En l'absence de ce modalisateur, il n'est pas toujours facile de comprendre comment procède la gradation éventuelle. En réalité, c'est le lecteur qui infère une gradation de la disposition des unités, en associant la dernière position de la phrase ou du segment à une position-clé investie d'un potentiel signifiant.

[22] Elles ont un capiteux, un charme, un je ne sais quoi tout particulier. [...] Comme il [cet œil de femme] trouble, envahit, possède, domine! (« Au printemps »: 159161)

La première suite se termine par une imprécision volontaire, qui sonne comme la réalisation prototypique du pouvoir de séduction d'une femme sur un homme, à la fois envoûtant et irrationnel. La suite des quatre verbes est sans doute plus problématique : elle manifeste certes le travail d'ajustement des mots aux choses mais, si on suppose que la place de chacun des éléments n'est pas interchangeable, comment la justifier? Le lecteur peut percevoir l'émergence progressive d'un sème d'abord absent, celui de la supériorité morale de la femme qui lui assure une puissance souveraine sur l'homme. Des relations se construisent entre les mots dans l'énoncé, comme si le verbe « domine » était ici l'aboutissement du long processus de séduction féminin. Un réseau s'esquisse qui ouvre à l'ensemble de la nouvelle, et de l'imaginaire maupassantien. Les 
reformulations offrent aussi une entrée dans un imaginaire, parce qu'elles proposent une approche du monde qui procède par reconfigurations successives.

La reformulation oriente l'organisation phrastique; elle peut aussi servir à l'organisation des séquences.

\subsection{Organisation séquentielle}

La reformulation prend place dans un réseau lexical et sémantique par des fonctions particulières.

Elle est synthétisante dans les exemples suivants :

[23] Toutes les bêtes, grenouilles et crapauds, ces chanteurs nocturnes des marécages, se taisaient. («Sur l'eau » : 74)

"Ces chanteurs nocturnes des marécages » fonctionne comme une reformulation qui subsume les deux noms cités auparavant. Les expansions du substantif jouent dans le calcul ou l'inférence qui permet de mettre en relation les différents syntagmes et noms qui se succèdent.

[24] Il la retrouvait dans mille circonstances oubliées: avec des physionomies effacées, ses gestes, ses intonations, ses habitudes, ses manies, ses colères, les plis de sa figure, les mouvements de ses doigts maigres, toutes ses attitudes familières qu'elle n'aurait plus. («En famille» : 116)

Le dernier segment de l'exemple [24], en clôture de phrase, remplit ce rôle de reformulation cohésive, par la présence de l'indéfini de la totalité «toutes » qui s'associe à un substantif au sens plus vague que les précédents et qu'on peut interpréter comme englobant. Ce groupe illustre un cas de reformulation assimilable à l'anaphore résomptive ou synthétisante. Les groupes qui le précèdent anticipent le syntagme « toutes ses attitudes familières ». L'évocation du souvenir du personnage se déplie à la faveur de syntagmes successifs qui en énoncent comme des éclats dispersés que seule réunit la mémoire affective du «il». Comme les éléments hétéroclites sont ainsi rassemblés, une reformulation globalisante assure, sur le plan syntaxique, l'unité de la séquence.

Un autre exemple est particulier en ce que la reformulation synthétisante se trouve avoir une portée à la fois anaphorique et cataphorique, prise entre deux énumérations successives.

[25] Mélange de calicots, de cabotins, d'infimes journalistes, de gentilshommes en curatelle, de boursicotiers véreux, de noceurs tarés, de vieux viveurs pourris ; cohue interlope de tous les êtres suspects, à moitié connus, à moitié perdus, à moitié salués,

à moitié déshonorés, filous, fripons, procureurs de femmes, chevaliers d'industrie à

l'allure digne. (« La Femme de Paul » : 168)

Le syntagme «cohue interlope de tous les êtres suspects» se distingue comme reformulation synthétisante par le sens globalisant du substantif qui subsume l'énumération à sa gauche et à sa droite. Le syntagme totalisant «tous les êtres suspects » se déplie en extension au fil des substantifs juxtaposés en parataxe asyndétique $^{17}$. 
La reformulation conduit à une lecture réticulaire du texte en esquissant des séquences construites sur des réseaux lexicaux. Elle intervient à un autre palier textuel, celui de la nouvelle tout entière dans un processus qu'on pourrait appeler cette fois spéculaire.

\subsection{Reformulation thématique et parabolique}

La reformulation est un mode de création et de réalisation de la textualité. «La femme de Paul » fournit un exemple de déploiement d'un substantif, le mot « tare », au travers de reformulations successives qui finissent par constituer le paragraphe tout entier :

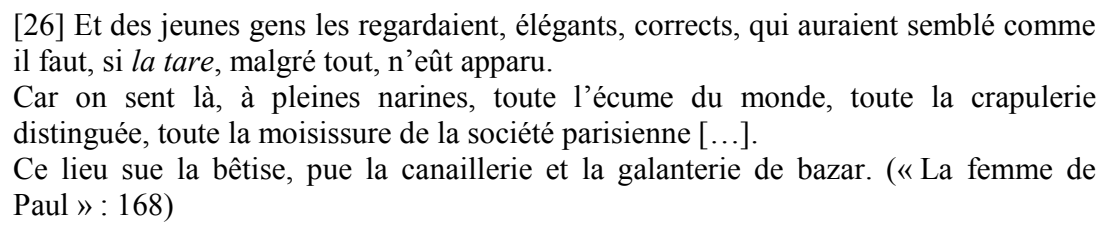

[26] Et des jeunes gens les regardaient, élégants, corrects, qui auraient semblé comme il faut, si la tare, malgré tout, n'eût apparu.

Car on sent là, à pleines narines, toute l'écume du monde, toute la crapulerie distinguée, toute la moisissure de la société parisienne [...].

Ce lieu sue la bêtise, pue la canaillerie et la galanterie de bazar. (« La femme de Paul» : 168)

Le palier maximal de la reformulation pourrait être réalisé dans la reformulation sémique et le concept d'isotopie. L'ensemble du paragraphe reprend les sèmes négatifs du mot « tare » et les déplie au gré de variantes hyponymiques, métaphoriques ou non : «l'écume du monde, la crapulerie, la moisissure, la bêtise, la canaillerie, la galanterie de bazar ».

Enfin, si on veut bien admettre ce passage au niveau macrostructural du texte, la reformulation peut être thématique et engager une reformulation qu'on pourrait appeler spéculaire : c'est le cas de l'épisode de la pêche du poisson dans « La femme de Paul » - reformulation parabolique de l'amour douloureux de Paul. Toute la scène de pêche est placée sous le point de vue du personnage ; la lecture parabolique est encouragée par le texte même qui multiplie les indices d'équivalence; l'analogie est perçue par le personnage même :

\section{Il se mit à tirer, et tout le gosier saignant de la bête sortit avec un paquet d'entrailles. Et Paul frémit, déchiré lui-même jusqu'au cœur ; il lui sembla que cet hameçon c'était son amour, et que, s'il fallait l'arracher, tout ce qu'il avait dans la poitrine sortirait ainsi au bout d'un fer recourbé. (« La femme de Paul» : 174)}

Le transfert de l'abstrait au concret est fréquent dans les contes. Ici, le cœur, siège métaphorique de l'amour, est remotivé comme organe vital; le sentiment de l'amour devient viscéral. La pêche, activité masculine par excellence dans les contes, est décrite ici comme exercice prédateur; le rapprochement avec le sentiment de l'amour éclaire l'imaginaire de Maupassant: l'amour est vu, lui aussi, comme un piège, aliénant et destructeur. La reformulation peut être dite spéculaire au sens où une scène, a priori indépendante de l'intrigue principale, reflète au contraire le nœud de l'intrigue même. La mise en scène énonciative permet d'établir l'équivalence entre les deux.

\section{Conclusion}

Pour que deux unités soient en relation de reformulation, l'unicité du référent a été démontrée comme critère nécessaire. Les unités peuvent y renvoyer successivement ou alors se partager les rôles entre une fonction de référenciation et une fonction 
commentative. Dans le premier cas, lorsque la fonction de référenciation est assurée successivement par les unités juxtaposées, la reformulation permet une recatégorisation du référent ; elle sera dite (re)dénominative. Dans le second cas, un lien de dépendance syntaxique de type appositif assure la cohésion des unités; la reformulation est alors d'ordre métalinguistique.

Dans les deux situations cependant, de dépendance ou d'autonomie syntaxique, subsiste, par le fait même de l'insertion dans la chaîne du discours, une interdépendance entre les items juxtaposés, qui en contraint l'agencement. La proximité sémantique des items, décrite en langue par le processus de synonymie par exemple, se trouve réactivée en discours par leur juxtaposition ou bien c'est la construction en série même qui induit le rapprochement sémantique; le lecteur est invité à reconstruire des réseaux lexicaux que le texte rapproche dans une suite linéaire orientée vers le point final d'une phrase. La lecture n'est plus seulement linéaire mais tabulaire et réticulaire. La reformulation organise la progression phrastique comme l'organisation des séquences textuelles. Ce faisant, c'est un travail sur le lexique qui est fait qui peut révéler l'imaginaire d'un écrivain par les nouvelles connexions qu'il établit entre les mots, les phrases, les paragraphes. Le cas limite de la reformulation, si on admet cette extrapolation possible de la phrase au texte, dessine une textualité spéculaire, qui procède là encore par étagement: quand des épisodes sont insérés dans la trame narrative et font écho à d'autres épisodes de l'histoire sur le mode parabolique. Le lecteur doit percevoir le reflet, la reformulation imagée d'un événement, et construire un nouveau réseau signifiant par ce jeu de miroirs.

Quel que soit le palier où elle s'exerce, au niveau sémantique, la reformulation peut procéder à la substitution d'un syntagme au précédent, dans un but d'ajustement des mots aux choses. Elle est alors à appréhender en termes de rectification, reposant sur cette stratégie d'effacement. Elle peut, au contraire, permettre l'empilement des items, sans que l'un soit donné comme "meilleur » que l'autre; aucune hiérarchie cognitive n'est à établir entre eux. Dans l'espace de la page et dans la mémoire du lecteur, toutes les composantes restent simultanément présentes. La reformulation, si fréquente dans les contes de La Maison Tellier, propose une vision démultipliée du référent ou plutôt des perceptions multiples du réel. C'est une image plurielle de la réalité qui se réalise, en particulier parce qu'elle repose sur des variations de points de vue. La réalité se construit par fragments successifs, tout comme le font les différentes nouvelles qui composent un recueil. Chacune succède à la précédente pour construire une image du réel, qui répond à la vision du conteur; chaque recueil de nouvelles même répond à un autre. Ces structures reformulatives pourraient être données comme modèle discursif des représentations, seules approches possibles du réel par le conteur.

\section{Bibliographie}

Adam J.-M. (2001). Les textes, types et prototypes. Récit, description, argumentation, explication et dialogue. Paris : Nathan.

Adam J.-M. (1990). Éléments de linguistique textuelle. Liège : Mardaga.

Blanche-Benveniste Cl., Caddéo S. (2000). "Préliminaires à une étude de l'apposition dans la langue parlée ». Langue française, 125, p. 60-70. 
Charaudeau P., Maingueneau D. (2002). Dictionnaire d'analyse du discours. Paris : Seuil.

Fuchs C. (1994). Paraphrase et énonciation. Paris : Ophrys.

Fuchs C. (1983). «La paraphrase linguistique : équivalence, synonymie ou reformulation? ». Le Français dans le monde, 178, p. 129-132.

Fuchs C. (1982a). La Paraphrase. Paris : PUF.

Fuchs C. (1982b). « La paraphrase entre la langue et le discours ». Langue française, 53, p. 22-33.

Gardes-Tamine J. (2011). Au cœur du langage, la métaphore. Paris : Champion.

Kleiber G. (2009). « La synonymie - «identité de sens » n'est pas un mythe ». Pratiques, 141-2, p. 9-25.

Langue française (1987). La reformulation du sens dans le discours, 73.

Le Bot M.-Cl., Schuwer M., Richard É. (2008). La reformulation : marqueurs linguistiques, stratégies énonciatives. Rennes : PUR.

Martin R. (1976). Inférence, antonymie et paraphrase. Paris : Klincksieck.

Murat M., Cartier-Bresson B. (1987). « C'est-à-dire ou la reprise interprétative ». Langue française, 73, p. 5-15.

Neveu F. (2004). Dictionnaire des sciences du langage. Paris : Colin.

Noailly M. (2000). «Apposition, coordination, reformulation dans les suites de deux GN juxtaposés ». Langue française, 125 , p. 46-59.

Rastier F. (2001). Arts et sciences du texte. Paris, PUF.

Rossari C. (1990). « Pour une typologie des opérations de reformulation ». Cahiers de Linguistique Française, 11, p. 345-359.

Rossari C. (1997). Les opérations de reformulation. Bern, Berlin, Frankfurt/M., New York, Paris, Wien : Peter Lang.

Roulet E. (1987). «Complétude interactive et mouvements discursifs ». Cahiers de Linguistique Française, 8, p. 111140.

Steuckardt A., Niklas-Salminen A. (2005). Les marqueurs de glose. Aix-en-Provence : PUP.

Vial A. (1954). Guy de Maupassant et l'art du roman. Paris : Nizet.

${ }^{1}$ Voir par exemple A. Steuckardt (2005). «Les marqueurs formés sur dire » p. 51-65 in A. Steuckardt et A. NiklasSalminen (2005) ou E. Khatchatourian (2008). "Les marqueurs de reformulation formés à partir du verbe dire », p. 19-23, in Le Bot M.-Cl., Schuwer M., Richard É. (2008).

2 Le programme de l'Agrégation de Lettres Modernes 2012, a donné l'occasion de relire ces contes et d'observer cette récurrence susceptible d'être interprétée comme une constante non aléatoire de l'écriture de ces textes.

${ }^{3}$ Le référent peut être appelé denotatum dans le triangle sémiotique de Odgen et Richard.

${ }^{4}$ On note une variation syntaxique dans la succession : dans le premier groupe, seul « les joues » assure la fonction de complément d'objet direct tandis qu'une relation d'ordre prédicatif ou attributif est instaurée entre les adjectifs « rouges », « pleines » et le syntagme nominal « les joues ».

${ }^{5}$ Dans les citations, les italiques soulignent les éléments qui sont commentés.

${ }^{6}$ Bikialo S. (2005). «De la reformulation à la glose : l'exemple de ou plutôt » p. 145-158, in A. Steuckardt et A. Niklas-Salminen (2005) : 156.

${ }^{7}$ Il sera question ici de la paraphrase dans sa dimension discursive - qui repose sur les reformulations d'un segment textuel dans un discours - et non pas de l'approche linguistique ou pragmatique.

${ }^{8}$ P. Charaudeau, D. Maingueneau (2002) : 417. Ou encore F. Neveu (2004) : 215 : «On appelle paraphrase la relation d'équivalence sémantique établie entre deux segments linguistiques ».

${ }^{9}$ C. Fuchs (1982a) : 57. « Les divers types de sémantismes et de paraphrases ».

${ }^{10}$ Voir C. Fuchs (1982a) pour la distinction entre l'identité de sens et l'équivalence de sens. L'identité de sens est en effet purement théorique, ce qui fait préférer la notion d'équivalence de sens ; cependant, au niveau pragmatique, le locuteur donne l'énoncé alternatif ou paraphrastique comme identique à l'énoncé premier.

${ }^{11}$ C. Fuchs (1996) : 53 et C. Fuchs (1982a) : $55:$ : La paraphrase constitue une relation d'équivalence sémantique en langue, qui se fonde sur l'existence d'un noyau commun (une sorte de «signifié de base» de la phrase correspondant peu ou prou au schéma propositionnel asserté) sur lequel viennent se greffer des sémantismes différentiels qui modulent diversement ce noyau de départ (des sortes de «signifiés secondaires » non pertinents pour l'établissement de la relation de paraphrase) ».

${ }^{12}$ Comme G. Mounin, B. Pottier, E. Coseriu.

${ }^{13}$ R. Martin (1976) : 113 distingue la synonymie absolue lorsque les items sont commutables dans tout contexte de la synonymie partielle dans le cas contraire.

${ }^{14}$ Voir aussi J. Tamine (2011) : 157

15 «Est virtuel tout élément qui est latent dans la mémoire associative du sujet parlant et dont l'actualisation est liée aux facteurs variables des circonstances de la communication ». [B. Pottier (1974). Linguistique générale. Théorie et description. Paris : Klincksieck, § 64 et 69, cité par Martin (1976) : 71]. 
${ }^{16}$ Le syntagme a été suffisamment étudié pour qu'une simple allusion suffise ici. Voir J.-M. Viprey (2006). «Un de ces [syntagmes] qui...». Corpus, 5, p. 159-214 et É. Bordas (1997). Balzac, discours et détours, Toulouse: Presses universitaires du Mirail ainsi que É. Bordas (2001). "Un stylème dix-neuviémiste. Le déterminant discontinu un de ces...qui ». L'Information grammaticale, 90, p. 32-43.

${ }^{17}$ Des cas attendus d'emploi anaphorique du démonstratif « cette » endossent aussi ce rôle de reformulation :

«Mais son image brusquement l'envahit, et il l'aperçut dans sa pensée quand elle s'éveillait au matin, dans leur lit tiède, se pressait câline contre lui, jetant ses bras à son cou, avec ses cheveux répandus, un peu mêlés sur le front, avec ses yeux fermés encore et ses lèvres ouvertes pour le premier baiser; et le souvenir subit de cette caresse matinale l'emplit d'un regret frénétique et d'un désir forcené. » (« La Femme de Paul » : 181).

Le syntagme démonstratif anaphorise de manière plus incertaine, soit le dernier syntagme de la liste, soit l'ensemble de l'énumération qui précède. Cet exemple excède cependant les limites qui ont été fixées pour ce travail en ce sens que la reformulation synthétisante n'est pas en construction paractatique par rapport aux termes support. 